\title{
SCIENCES ET TECHNIQUES
}

Jean-Yves Goffi, La Philosophie de la technique. Paris, P.U.F., 1988. 11,5 × 17,5, 127 p. («Que sais-je?", 2405).

La réussite d'un " Que sais-je ? " portant sur un domaine aussi vaste que celui de la philosophie de la technique mérite assez d'être soulignée, tant il est difficile d'être aussi précis et érudit que J.-Y. Goffi l'a été en cent vingt-sept pages. Ainsi, les légères critiques que nous inspire ce texte sont-elles frappées d'entrée de jeu d'insignifiance, quand ce n'est pas d'injustice. Mais la richesse du détail des analyses n'empêche pas plusieurs interrogations sur le projet général poursuivi par l'auteur.

Sous le titre de La Philosophie de la technique, le livre offre en réalité un classement des grandes philosophies de la technique du passé et du présent. De chacune, l'auteur trace la logique interne avec une précision étonnante; chacune est subtilement relativisée par une fine critique ou un trait d'ironie. La prétention de l'ouvrage n'est donc pas simplement doxographique.

Mais voilà bien le point : la galerie des illusions étant passée en revue et ayant permis de dégager les catégories majeures de la pensée de la technique, le lecteur en attend le dépassement, soit sous la forme d'une ultime figure qui équilibrerait différemment le jeu des catègories, soit par une interprétation de style psychanalytique qui rendrait compte en particulier de la « technophobie ", soit enfin par une compréhension des raisons pour lesquelles une culture, prise à divers moments de son histoire produit - jusque dans la tête des philosophes - des représentations fallacieuses de son système de techniques liées à une tonalité généralement hostile à son objet. Les hommes - y compris les philosophes - ne pensent pas leurs techniques comme ils les vivent. La tâche de la philosophie en ce domaine ne peut-elle être d'établir les relations qui existent entre la pratique réelle de ces techniques et les représentations qui l'expriment et peut-être la masquent? Sa mise en œuvre impliquerait alors autant une attention vigilante aux figures par lesquelles la conscience prétend dire la réalité même du phénomène, qu'une vue plus radicalement critique à l'égard des diverses philosophies produites dans le domaine.

Il semble que l'auteur ne tranche pas entre les points de vue les plus opposés qu'il décrit et qu'il les traite comme des interprétations possibles de la nature des techniques. S'il affirme «le caractère essentiellement historique des techniques ", il n'en exclut pourtant pas la possibilité d'une conception vitaliste, quoiqu'il en dénonce çà et là les illusions. On le voit revenir à plusieurs reprises sur une conception darwinienne de l'évolution des techniques, quitte à prendre ses distances à son égard. Il diagnostique une «technophobie contemporaine » et 
semble donc accepter son caractère historique, mais il croit aussi à une « technophobie éternelle ". Cette conception irénique tient sans doute à l'hétérogénéité du phénomène étudié ; elle tient aussi - et la clé nous en est donnée dans le chapitre de conclusion - aux convictions libérales de l'auteur quant aux vertus de l'économie de marché qui, par sa régulation impersonnelle des activités humaines, s'opposerait efficacement aux projets technologiques les plus fantasques des particuliers ou de leurs gouvernements. Les projets culturels livrés à la concurrence, soit à la sélection réciproque, produisent l'effet d'une nouvelle nature qui évolue sur un rythme propre et selon des lois qui ne sont celles d'aucune conscience particulière.

Saisissant qu'elle n'est pas au principe du mouvement technologique, la conscience n'aurait pas intérêt à feindre de s'y placer et ne pourrait avoir confiance dans ses capacités à en prendre la direction. La volonté délibérée d'agir dans le domaine pour prétendre y mettre de l'ordre y créerait plus d'inconvénients que d'avantages et provoquerait, dans l'état actuel de la technologie, les conditions d'un «totalitarisme ». L'argument est bien connu depuis Hume qui vénérait les vertus de l'inconscience dans le domaine des fondements de la politique et ironisait lui-même sur les paradoxes de cette étrange position philosophique. Peut-on le réactiver sans précautions dans le domaine des techniques où l'inconscience aurait aussi ses vertus? Ne risquons-nous pas de tomber, en préconisant cette abstinence pratique, dans la contradiction qui consiste à vouloir dénoncer dans ses racines la "technophobie" en se privant des moyens de le faire effectivement? Ceux qui jouèrent au jeu de la "bonne inconscience" au $\mathrm{XVIII}^{\mathrm{e}}$ siècle ne laissèrent pas d'être joués à leur tour et crurent parfois déceler le meilleur équilibre politique de l'Europe dans les conditions mêmes de la Révolution française.

Certes l'auteur indique lui-mème une limite de son argument et n'entend pas que la construction des centrales nucléaires relève dans le même sens de la sélection naturelle que celle des bateaux de pêche - chers à Alain qui a pu en trouver l'exemple chez Hume et chez Mandeville. Mais il est difficile de partager sa foi dans l'autorégulation du marché, comme si ce système n'était jamais en crise et comme si le « totalitarisme " ne pouvait être fabriqué par ces crises mêmes. On doutera aussi de la définition si peu dialectique du marché comme « la toutepuissance de l'acheteur sur le vendeur " ; n'est-ce pas, pour le moins, surestimer la sanction que le premier exerce sur le second? On s'étonnera de voir régler en un tournemain le problème éthique posé par les techniques; suggère-t-on par là, à la façon de certains textes de Hume, que la sélection des valeurs morales s'effectue, comme pour n'importe quelle autre valeur culturelle, par une sorte de concurrence? Auquel cas il faudrait aussi confier au "libre jeu " du marché les conditions de leur production. Que le marché soit indiscutablement une dimension du principe de réalité des valeurs techniques n'autorise tout de même pas à le traiter comme l'ultime instance de tous les jugements que l'on peut porter à leur égard.

Enfin le débat sur la politique technologique n'est-il pas abusivement simplifié lorsque se trouve opposé un libéralisme économique, dont la pureté serait garantie et réglée par la loi, au « totalitarisme » (qu'on devrait toujours prendre 
la peine de définir, tant le terme est ambigu)? En présentant l'impersonnalité du premier comme un avantage, l'auteur ne laisse-t-il pas dangereusement penser que la personnalité serait l'apanage du second?

Le livre de J.-Y. Goffi pose en ce sens plus de problèmes qu'il n'en résout; nous trouvons là son principal mérite et la pleine justification de sa place dans cette collection encyclopédique des Presses universitaires de France.

Jean-Pierre Cléro.

Héron d'AleXandrie, Les Mécaniques ou l'Élévateur des corps lourds. Éd. et trad. de l'arabe Bernard CARRA DE VAUX, d'après le texte de Qustâ ibn Lûqâ, introd. Donald R. HiLl, commentaires A. G. DraChmanN. Paris, Les Belles Lettres, 1988. $16 \times 24,305-115 \mathrm{p}$, texte en arabe précédé de la trad. française, commentaires en anglais, reprod. en fac-sim. de l'éd. de 1894 pour le texte arabe et la trad. de Carra de Vaux (« Sciences et philosophie arabes. Études et reprises ").

Cet ouvrage est une sorte de manuel théorique et pratique, à l'usage de techniciens travaillant sur des chantiers de construction. Il a été composé par Héron d'Alexandrie au premier siècle de notre ère, et cet auteur s'y montre explicitement dépendant de toute la mécanique grecque qui l'a précédé, ce qui en fait pour nous une source très importante pour la connaissance de cette tradition, dont peu de témoins sont conservés.

Le texte des "Mécaniques de Héron" est perdu dans sa langue grecque originale, mais nous est transmis dans une version arabe faite au IX $x^{\mathfrak{e}}$ siècle par Qustâ ibn Lûqâ al-Ba'albakî, célèbre traducteur de textes scientifiques du grec à l'arabe. Ce texte arabe avait été édité, avec traduction française, par B. Carra de Vaux, dans le Journal Asiatique en 1893 et 1894 . Le travail d'établissement du texte arabe a ensuite été refait, accompagné d'une traduction allemande, par L. Nix (Herons von Alexandria Mechanik und Katoptrik, hrsg. und übersetzt von L. Nix und W. Schmidt, Opera quae supersunt omnia, vol. 2, fasc. 1, Leipzig, 1900). Mais cette reprise par L. Nix de l'établissement du texte, bien que faite sur quatre manuscrits au.lieu d'un seul pour Carra de Vaux, n'apporte pas d'éléments vraiment nouveaux pour la connaissance du contenu de l'ouvrage comme tel.

Le choix de l'éditeur de l'ouvrage présenté ici est d'avoir réimprimé l'édition et la traduction de Carra de Vaux; elles sont précédées d'une préface par D. Hill, très bon connaisseur de la mécanique ancienne, qui fait le point sur ce que l'on peut savoir de Héron d'Alexandrie, de ses sources, de ses œuvres et de leur influence sur la mécanique arabe; et l'ouvrage se termine sur des commentaires en anglais par A.G. Drachmann, sous forme de "notes complémentaires" référées à la page et à la ligne du texte, où ce dernier auteur critique l'établissement du texte arabe et sa traduction, à la fois chez Carra de Vaux et chez Nix en comparant les travaux de ces deux auteurs, et commente en détail le travail de 
Héron, en refaisant toutes les figures et en replaçant chacun des procédés décrits dans l'histoire de la technique ancienne.

Cet ensemble permet ainsi d'avoir de nouveau accès à cet ouvrage dans de très bonnes conditions, avec toutes les garanties possibles pour une connaissance précise du texte et de son contenu. Ce sera un auxiliaire précieux pour les historiens de la technique, ou, plus généralement, pour les historiens des sciences.

Régis MORELON.

David S. LANDES, L'Heure qu'il est: les horloges, la mesure du temps et la formation du monde moderne. Trad. de l'anglais Pierre-Emmanuel DAUZAT, Louis Évrard. Paris, Gallimard, 1987. $14 \times 21,632$ p., 157 ill. dt 24 pl. (« Bibliothèque illustrée des Histoires »).

Voici l'un des plus grands livres d'histoire de ces dernières années. Le sujet, l'ampleur de vue, la précision des sources et la qualité de l'écriture et de la traduction, tout est remarquable. De plus, cette deuxième édition est revue et corrigée et donc meilleure que l'original (Harvard U.P., 1983). Histoire culturelle, histoire des sciences et de la technique et, enfin, histoire économique et sociale, une série de questions structure le livre: pourquoi l'horloge mécanique fut-elle inventée en Europe, comme devint-elle de plus en plus sophistiquée, qui l'a construite et pourquoi?

En réalité, c'est de la respiration des hommes dans le temps dont il est question au centre de ces pages, dont on ne dira jamais assez la finesse et l'érudition. De l'Asie au Saint-Empire, des académies aux plus petits colporteurs, des fins lettrés aux managers japonais, c'est l'occasion d'étudier la naissance, la maturité et l'obsolescence de neuf siècles d'une branche de l'industrie manufacturière. Si la Chine n'avait nulle besoin de connaitre le temps avec précision, on n'imagine pas le monachisme chrétien sans une mesure précise du temps. La montre, qui fait son apparition dans le dernier quart du $\mathrm{Xv}^{e}$ siècle, permet de " garder " le temps, ce qui est au moins aussi important que de l'établir. La trotteuse centrale avec cadran indiquant les fractions de seconde apparait vers 1770 . C'est le temps à la portée de tous, et d'une précision qui permettra de conquérir le monde. Les chemins de fer américains du $\mathrm{XIX}^{\mathrm{e}}$ siècle sauront aussi l'utiliser! Mais la géographie de cette technologie n'est pas absente puisque les centres allemands, français, anglais sont présentés avant d'atteindre la Suisse qui bénéficia peu à peu d'un monopole du $\mathrm{XVIII}^{\mathrm{e}}$ au $\mathrm{XX}^{\mathrm{e}}$ siècle. L'histoire culturelle et technologique devient également commerciale. Unification du globe, victoire sur le temps - dont on ne sait pas toujours bien ce qu'il est mais que l'on sait au moins mesurer -, le triomphe des manufactures suisses est attaqué par le Japon ainsi que par la montre à quartz dont le premier modèle date de 1928 aux U.S.A. Tout est bouleversé et les paramètres industriels changent complètement. C'est aussi l'histoire de l'Occident moderne jusqu'à nos jours que ce livre magnifique - l'iconographie est superbe 
- retrace. Un champ entier de recherche est désormais ouvert et balisé où les découvertes ne manqueront pas. Ainsi en témoigne, par exemple, un récent article de Vincent Chenille, "L'horlogerie parisienne : innovation technique et malthusianisme économique au XIX ${ }^{\mathrm{e}}$ siècle " (Sources. Travaux historiques, 19, 1989, p. 29-36).

Dominique Bourel.

Jean Picard et les débuts de l'astronomie de précision au xvIr siècle. Actes du colloque du tricentenaire, éd. par Guy PICOLET. Paris, Éd. du C.N.R.S., 1987. $15,6 \times 24,382$ p. ( (Centre régional de publication de Paris »).

Guy Picolet a réuni sous ce titre les communications présentées au colloque du tricentenaire de Jean Picard, les 12 et 13 octobre 1982. Les historiens et tous les curieux de l'histoire des sciences dans ce grand siècle que fut le $\mathrm{XVII}^{\mathrm{e}}$ trouveront dans ce recueil une très riche documentation.

La première partie traite de la biographie du savant. Sur ses origines - qui étaient fort mal connues -, Guy Picolet s'est livré à une enquête approfondie qui nous révèle, en particulier, comment le modeste Jean Picard, après ses études au séminaire, a pu devenir un savant de premier plan. John W. Olmsted, dans le seul article en anglais du recueil, étudie les conditions de la nomination de Picard à la toute nouvelle Académie des sciences (1666-1667).

La deuxième partie réunit les contributions sur l'œuvre proprement astronomique de Picard. Elle se situe à l'époque où, après les extraordinaires données obtenues par Tycho Brahe sans instrument d'optique, l'invention de la lunette, de l'oculaire convergent par Huygens et celui du micromètre vont permettre un capital pas en avant dans la précision des mesures. Ce qu'étudie Jacques Lévy, "Picard créateur de l'astrométrie moderne " et Suzanne Débarbat qui analyse « la qualité des observations astronomiques de Picard ", tous articles qui intéresseront d'abord les spécialistes. Au contraire, le texte de Kurt M. Pedersen, « Une mission astronomique de Jean Picard: le voyage d'Uraniborg ", passionnera tous les lecteurs. On peut dire que cette mission au Danemark est une première : un voyage lointain à mission scientifique; il s'agissait de faire des mesures précises sur les lieux du château d'où Tycho Brahe avait observé, afin que ses données puissent être raccordées avec celles des astronomes de l'Observatoire de Paris. On sait que Picard embaucha à cette occasion le jeune Olaüs Römer et qu'il le ramena avec lui à Paris où il devait réussir la première mesure de la vitesse de la lumière. Bel exemple des effets heureux de la coopération internationale.

Leçon d'une autre sorte donnée par le récit de Kurt Pedersen, Picard avait rapporté dans ses bagages les manuscrits de Tycho Brahe qui lui avaient été confiés à fin d'édition. Les crédits manquèrent ou l'administration française tergiversa, bref, Picard mourut sans avoir pu réaliser ce travail essentiel pour l'histoire de l'astronomie. On sait que les manuscrits durent être restitués au 
Danemark où, comble de malchance, ils disparurent dans un incendie. On mesure ici les conséquences dramatiques du retard d'un simple travail d'édition.

Picard fut également pionnier dans les mesures géodésiques. C'était une autre conséquence de l'invention de la lunette : les mesures de triangulation pouvaient atteindre une précision inégalée. René Taton rappelle ici l'importance de la mesure de la Terre puisque le résultat ne fut pas pour rien dans le développement des idées de Newton sur la gravitation universelle. Picard fut aussi cartographe et c'est l'occasion pour nous de signaler la qualité et l'intérêt des documents originaux reproduits ici : la grande carte de France montre l'importance des corrections apportées par Picard aux tracés fort fantaisistes qui avaient cours avant lui.

Picard fut également intéressé par les travaux d'alimentation en eau du palais de Versailles, ce qui l'obligea à renouveler les méthodes de nivellement.

Les activités scientifiques de Picard l'amènent à s'intéresser à l'optique, à la construction des cadrans solaires mais aussi, et c'est le plus intéressant pour nous, à la définition des unités de longueur. Pierre Costabel étudie les tentatives (infructueuses) de Picard pour définir l'unité de longueur par la longueur du pendule battant la seconde. Mais c'est l'époque où Richer, à Cayenne, s'aperçoit que la longueur de ce pendule battant la seconde dépend de la latitude géographique. Au fond, on en arrive à regretter que Picard soit mort trop tôt, il n'a pas connu les idées de Newton qui auraient sans aucun doute orienté ses recherches.

Le colloque du tricentenaire avait été une réussite que la publication de ce recueil parachève. Jean Picard est un exemple de savant, modeste dans sa personne, singulièrement attachant par ses travaux qui le situent bien au centre de l'activité scientifique de son temps.

Gilbert WALUSINSKI.

Josef Konvirz, Cartography in France. 1660-1848. Science, Engineering, and Statecraft. Chicago/Londres, The University of Chicago Press, 1987. $18 \times$ 25,5, 194 p., ill., index.

La publication de l'ouvrage de J. Konvitz est l'occasion de souligner la faiblesse de la recherche française en histoire de la cartographie. Quelques articles dispersés ou le catalogue de l'exposition "Espace français" des Archives nationales ne peuvent masquer une curiosité bien modérée à l'égard des cartes anciennes. Sources parmi d'autres sources, les historiens n'y puisent souvent que quelques informations ponctuelles. Les géographes, quant à eux, négligent l'histoire d'un instrument pourtant fondamental de l'analyse et de la maitrise de l'espace.

Le retard pris dans ce domaine est aussi un retard théorique : la carte " poignée de faits ", l'histoire esthétisante de la cartographie, le darwinisme cartographique (les cartes anciennes étant jugées dans un cadre évolutionniste, leurs progrès et gains de précision rapportés aux modèles modernes) sont autant de conceptions que la recherche, notamment anglo-saxonne, invite à corriger, à dépasser. 
Avec le livre de Konvitz, introduit par E. Le Roy Ladurie, la carte s'élève au rang d'objet à part entière du savoir historique, éclairé, selon une inversion du rapport traditionnel, d'autres histoires, notamment ici de celle des sciences et de la puissance publique.

Konvitz a conduit, pendant une année, des recherches approfondies dans des fonds d'archives qui témoignent utilement de l'activité cartographique française, de 1660 à 1848, bien qu'ils aient été jusqu'alors peu explorés dans cette optique (Archives du Génie, de l'École des ponts et chaussées, etc.). Son ouvrage met l'accent sur les relations entre la cartogáaphie et l'État français. Il s'ordonne autour de trois points principaux : la carte de France, dite de Cassini, le procédé de la courbe de niveau, et les cartes thématiques. L'auteur précise le rôle de la cartographie dans le domaine militaire, politique, économique, et met en valeur l'influence des cartes sur la perception et l'utilisation de l'espace. Le XVIIr siècle est privilégié, la France dominant alors la cartographie internationale, et proposant les innovations les plus importantes.

Après une période de "sous-développement » cartographique, sous Henri IV et Louis XIII, l'avancée est spectaculaire, grâce à l'impulsion donnée par l'État, ses ministres ou ses institutions. Konvitz nous renvoie l'image d'un État protéiforme, où se développent souvent concurremment les projets cartographiques de bureaucraties autonomes. Son travail reflète assez les cloisonnements administratifs de l'État : il apparaît plutôt comme une série d'essais organisés autour d'un projet et, parfois, d'une source particulière.

Parmi les sujets abordés, certains, comme la carte de Cassini ou la cartographie thématique, ont pu faire l'objet d'études antérieures. Konvitz les enrichit toutefois de documents inédits, et veille à mettre l'accent sur les facteurs politiques et surtout scientifiques externes à la cartographie. Ainsi, il évoque les relations entre les mesures géodésiques servant de base à la carte de Cassini et les théories newtoniennes sur les sphérö̈des, mentionnant au passage les expéditions organisées par l'Académie des sciences, en Suède et au Pérou, entre 1734 et 1744, pour mesurer la longueur d'un degré de longitude. Dans le même esprit, l'étude du cadastre avant 1799 s'accompagne d'un long développement sur la réforme du système de mesure.

Konvitz rend justice à bien des serviteurs de l'État, Colbert, le contrôleur général Orry, ou le directeur de l'École des ponts et chaussées, puis du service du cadastre, Gaspard-François de Prony. Il ne peut toutefois éviter de s'écarter de son propos initial, et d'évoquer le rôle cartographique d'un " secteur commercial ". L'indépendance du secteur privé et du secteur public ne peut être posée trop strictement : bien des entreprises cartographiques résultent d'une combinaison d'initiatives de l'un et de l'autre. Ainsi, après 1756, c'est une compagnie par actions qui relaie l'effort gouvernemental, et permet à Cassini de Thury de poursuivre les travaux de la carte de France. Par ailleurs, en matière de cartographie thématique, de 1780 à 1850 , les innovations doivent peu à l'État français, y compris dans le cas, évoqué par Konvitz, de la cartographie économique de Charles-Joseph Minard. Ce dernier, bien qu'issu des Ponts et chaussées, dessine de sa propre initiative ses cartes de production et de flux, une fois retraité.

L'étendue de la période d'étude, d'abord surprenante, doit être relativisée. 
Konvitz reconnaît avoir écarté certains thèmes, malgré leur rapport avec le fil conducteur qu'il s'était choisi. On le note avec quelque regret : il semble notamment qu'une analyse du rôle de l'État dans la cartographie sous le Consulat et l'Empire aurait été particulièrement féconde, eu égard à l'œuvre considérable accomplie alors par les ingénieurs géographes militaires.

Konvitz a certes commis quelques erreurs de détail, mais tout en ayant disposé d'un temps de recherche relativement court, il a pu exhumer des cuvres originales et méconnues, à l'image de ce que fit en son temps le Père de Dainville, infatigable découvreur de cartes. Il faut ainsi accorder dans cet ouvrage une attention toute particulière à l'histoire inédite de la constitution, par le ministère des Affaires étrangères, d'une collection de cartes destinées à servir lors des négociations diplomatiques sur les frontières, et du projet complémentaire d'un atlas de frontières, entrepris par le Bureau topographique pour la démarcation des limites, créé en 1775.

On ne peut qu'espérer que la contribution utile et originale de Konvitz ouvre la voie à d'autres études, parfois mentionnées incidemment par l'auteur. Le département des cartes et plans de la Bibliothèque nationale constitue un support exceptionnel pour de telles recherches, et bien d'autres archives cartographiques attendent leur inventeur. Konvitz lui-même devrait prochainement proposer une synthèse sur la cartographie du secteur public et le commerce privé des cartes, dans la monumentale History of Cartography dirigée par J. B. Harley et D. Woodward, dont le volume 1 est paru en 1987.

Gilles Palsky.

Dennis L. SEPPER, Goethe contra Newton. Polemics and the Projects for a New Science of Colour. Cambridge, Cambridge University Press, 1988. 15,8 $\times$ 23,5, XVI-222 p., bibliogr., index.

Commençant par un historique de l'intérêt éprouvé par Goethe pour l'étude scientifique de la couleur, le professeur Sepper montre comment cette ardente curiosité s'est développée progressivement à partir d'un malentendu fécond, d'une lecture hâtive des expériences décrites par Newton. Ignorant le fait que le newtonisme rendait parfaitement compte de phénomènes qu'il croyait observer pour la première fois, Goethe réalise avec le prisme une série d'expériences primordiales qui le conduisent à émettre des critiques fondées et à remarquer certaines limites inhérentes à la méthode et à la doctrine newtonienne, ce qui le rattache aussi bien aux principaux adversaires contemporains de Newton (Huygens, Hooke) qu'à quelques développements récents de l'expérimentation sur les couleurs (Ghercke, Edwin Land). Des Beiträge zur Optik de 1791-1792 à Zur Farbenlehre (1810), on assiste à l'élargissement des perspectives initiales et notamment à l'approfondissement d'une réflexion d'ensemble sur la science, tandis que le ton violemment polémique adopté par Goethe, empêche sa pensée de toucher son public potentiel, déjà largement acquis au newtonisme. En effet, 
le professeur Sepper, décrivant la diffusion de la connaissance scientifique en Allemagne au $\mathrm{XVII}^{\mathrm{e}}$ siècle, montre que les manuels les plus courants offraient une présentation vague et lacunaire des principaux aspects de la science newtonienne, ce qui suffirait à expliquer la déception de Goethe lorsqu'il s'intéressa à l'étude des couleurs.

Mais cette insatisfaction était plus profonde : Goethe rêvait d'une science qui, établie sur un fondement descriptif précis, sût rester fidèle à la totalité des phénomènes colorés, alors que Newton avait réduit la science des couleurs à n'être qu'une simple province de l'optique, théorie essentiellement physicomathématique. Élaborant dans les Beiträge zur Optik le concept fondamental de Ur-Phänomenon, (totalité phénoménale résultant de la synthèse de toutes les conditions nécessaires à son apparition), Goethe exige de la science qu'elle ne laisse de côté aucune des circonstances de l'expérience et reproche à Newton de réduire celle-ci au statut de simple illustration de la théorie. C'est le début de sa critique, qui trouvera son aboutissement dans Zur Farbenlehre, de la notion d'experimentum crucis. L'étude de la place de cette notion chez Newton et de sa critique par Goethe occupe une partie considérable de l'ouvrage de D. Sepper, qui se livre à une véritable relecture des textes canoniques de Newton (essentiellement la Lettre à la Royal Society du 6 février 1672). L'auteur, rappelant l'origine de la notion dans l'épistémologie baconienne, décrit le coup de force réalisé par Newton lorsque celui-ci, rompant avec la tradition empiriste antérieure, prétend donner à une expérience privilégiée, l'expérience cruciale, le statut non plus seulement de réfutation possible des théories adverses, mais encore de démonstration empirique de sa propre théorie. Pour Newton, décrire une expérience bien choisie, c'est tout de suite exhiber la théorie, sans recours aucun aux hypothèses. Paraphrasant Goethe, le professeur Sepper se situe ici dans le prolongement de critiques antérieures de la doctrine newtonienne, notamment celle de Paul Feyerabend '. Puisque l'une des cibles de la critique de Goethe est précisément l'excès d'abstraction qu'il reproche à la science de son temps, on ne s'étonnera pas que l'une des qualités majeures du livre soit de ne pas s'encombrer d'une technicité qui nuirait à son propos : le professeur Sepper, qui recommande à son lecteur de se munir d'un prisme, expose dans un court appendice les éléments les plus techniques des découvertes de Goethe. Plusieurs schémas très clairs achèvent de rendre la lecture confortable.

Observant le rôle majeur joué par le contraste de la lumière et de l'obscurité dans l'apparition du spectre des couleurs, Goethe en vient à reprocher à Newton d'analyser en termes de rayons un phénomène qui se présente à l'expérimentateur sous la forme d'une image (Bild), et ainsi de prétendre faire passer pour pure et simple description du visible, ce qui est déjà expression théorique du substrat invisible du phénomène. L'apport goethéen est double : retourner au fondement expérimental des théories, et à partir de l'observation attentive de ce fondement, désigner les déficiences de la théorie dominante. La faute commise par Newton

1. Paul Feyerabend, «Classical Empiricism », in R.E. Butrs, J.W. Davis, dirs, The Methodological Heritage of Newton, Toronto, Toronto University Press, 1970, p. 150-170. 
consiste, d'une part, à introduire le concept de rayon, ce que n'autorise nullement la seule observation du phénomène, d'autre part, à négliger toutes les circonstances qui le composent, en particulier le rôle joué aussi bien par la constitution physique et chimique du milieu de propagation de la lumière que par les phénomènes physiologiques et psychologiques à mettre au compte de l'observateur lui-mème.

Exhumant un courant hétérodoxe de la science des couleurs au XVIII siècle (Scherffer, R. W. Darwin, Guyot, Mauclerc, etc.), Goethe, qui élabore la notion de Vorstellungsart (façon de concevoir les phénomènes), prolonge sa réflexion dans la direction d'une politique ou, comme on voudra, d'une éthique de la science : la communauté des chercheurs, aussi bien que l'organisation de la science dans l'État, doivent garantir l'existence d'une pluralité de Vorstellungsarten (essentiellement le couple mécanisme/dynamisme), afin que la richesse problématique des phénomènes ne soit jamais perdue de vue et qu'aucune doctrine n'exerce sur la pratique scientifique l'hégémonie sclérosante qui fut celle du newtonisme dans le domaine de l'optique. Pour Goethe, témoin impuissant de la professionnalisation croissante de la science, la vérité scientifique, tâche infinie, ne se réduit pas à un ensemble de propositions; la science n'est ni méthode, ni langage, ni mathématique, mais une certaine activité de l'esprit humain qui rassemble certes ces éléments mais qui, conçue comme véhicule d'une formation (Bildung), doit conserver un lien dynamique avec son passé. L'histoire de la science devient pour Goethe une partie essentielle de la science mème.

Du seul point de vue des études newtoniennes, l'ouvrage de Dennis L. Sepper apporte déjà une contribution considérable à notre connaissance de la réception du newtonisme en Europe, après les ouvrages de Henry Guerlac, Paolo Casini, etc. ${ }^{2}$, dont il se différencie par son optique monographique. Il serait intéressant, à ce titre, de confronter cette étude avec ce que nous savons déjà de la place occupee par la science newtonienne dans la philosophie de Kant ${ }^{3}$ ou de Hegel. Mais en inscrivant son étude dans la lignée de travaux épistémologiques tels que ceux de Kuhn ou de Lakatos, l'auteur montre tout l'intérét que peut présenter pour la philosophie des sciences une lecture des travaux d'optique de Goethe. A condition de faire abstraction de la dimension polémique, historiquement nuisible, de ces travaux (le livre explore avec rigueur la genèse de cette démarche et les sentiments changeants de Goethe à son égard), il est tout à fait possible d'y découvrir une pensée de la science, à la fois comme théorie et comme pratique, aux accents souvent étrangement actuels.

Jean-François BAILLON.

2. Henry Guerlac, Newton on the Continent, Ithaca, N.Y., Cornell University Press, 1981 ; Paolo CaSinI, Newton e la coscienza europea, Bologne, Il Mulino, 1983 ; A. I. SABRA, Theories of Light from Descartes to Newton, Cambridge, Cambridge University Press, 1967, rééd. 1981 ; Michel BLAY, La Conceptualisation newtonienne des phénomènes de la couleur, Paris, Vrin, 1983.

3. Étude faite par François MARTY, in La Naissance de la métaphysique chez Kant, Paris, Beauchesne, 1980. 
Jean-Rodolphe Perronet, Construire des ponts au xvIr siècle. Liceuvre de $J$.R. Perronet. Prés. de Jean-Rodolphe Perronet par Michel YvoN. Paris, Presses de l'École nationale des ponts et chaussées, $1987.27 \times 37,340 \mathrm{p}$., pl.

Cet ouvrage de format grand in $-4^{\circ}$, illustré de magnifiques dessins et gravures, est tiré de la deuxième édition des Euvres de $M$. Perronet (première édition, 1782) et a été luxueusement réalisé par l'Association amicale des Ingénieurs anciens élèves de l'École nationale des ponts et chaussées, aux Presses de l'École sus-dite, 28, rue des Saints-Pères, 75007 Paris, avec l'appui de la Compagnie des autoroutes (COFIROUTE).

Cette réalisation s'inscrit dans la préparation du $250^{e}$ anniversaire de la fondation de l'École des ponts et chaussées, en 1997. C'est, en effet, en 1747 que JeanRodolphe Perronet reçut la triple mission de former, de construire et de diriger, mission qu'il remplit jusqu'à sa mort en 1794. Remarquable ingénieur, Perronet ne fut pas seulement le directeur d'une grande École, il fut l'animateur d'une œuvre considérable concernant les travaux publics sur les routes, les ponts et les canaux. Comme le souligne M. Michel Yvon, conservateur de la Bibliothèque de l'École nationale actuelle, Perronet a doté la France de Louis XV et de Louis XVI de moyens de communication marqués au signe de la perfection technique. Parmi ces moyens, les ponts tiennent une place importante et se distinguent par leur valeur artistique, "leur hardiesse et leur solidité ".

Cette dernière expression figure sous la plume de Perronet dans le « Discours préliminaire " reproduit dans le présent ouvrage. Elle est associée à l'affirmation que les travaux français " pourront un jour servir de modèles aux architectes des autres nations ». Et la nécessité d'une publication sur le sujet est rapportée au fait que « l'établissement des ponts exige, tant pour les fonder que pour les élever, des moyens qui disparaissent après leur entière construction ". C'est une remarque pertinente.

Aussi les descriptions et les admirables planches qui les illustrent font-elles une place à tous les détails de construction (pieux, cintres, piles, etc.). Mais, à la suite des chapitres où ces détails sont traités de manière strictement technique dans les cas particuliers où il a fallu les mettre en ouvre, l'édition originale contenait des mémoires scientifiques dont un seul - relatif à la méthode de cintrer et de décintrer les ponts - a été reproduit.

On peut regretter que cette édition ait été ainsi tronquée, mais le choix du mémoire conservé a été bon parce qu'il s'agit d'un texte significatif. C'est à l'usage que l'on s'est rendu compte que pour les arches de pont de grandes portées, les cintres s'affaissent après leur assemblage et pendant leur construction, et Perronet s'est attaché à évaluer par expérience ce que l'on doit attendre pour cet affaissement, puis à déterminer comment on doit ensuite modifier les épures qui servent aux charpentiers. Il n'y a donc pas lieu de compter sur un exposé mettant en application explicite des connaissances théoriques sur la statique des voûtes. On y trouve cependant l'assurance que l'appel à l'expérience était subordonné à une conception de recherche : faire en sorte que les pressions dues à la pesanteur se conjuguent sur les piliers. Et il est intéressant que la conclusion, dans le cas du 
pont de Neuilly, soit qu'avec une portée assez considérable la voûte prenne la forme d'un arc de cercle.

A prononcer le nom du pont de Neuilly, il faut ajouter que l'ouvrage, grâce à ses illustrations, est un véritable objet d'art relatif à des constructions qui, en d'autres lieux comme Mantes, Orléans, Sainte-Maxence, etc., voire SaintPétersbourg, sont elles-mêmes des œuvres d'art. Les éditeurs méritent la gratitude d'un large public cultivé.

Il semble seulement qu'ils ont omis inconsciemment une table des matières qui eût été fort utile, ainsi que la mention précise des coupures qu'ils ont faites en reproduisant seulement " les textes les plus significatifs ». L'avertissement à cet égard, discrètement inscrit dans une "Note de l'éditeur", échappera probablement à beaucoup de lecteurs. Comme leur échappera aussi l'explication de la fin du titre, à savoir " pour servir de complément à la nouvelle Architecture hydraulique ". Le célèbre ouvrage de Belidor, Architecture hydraulique, a eu une édition en 1788 pour une seconde partie et sous le nom de Belidor (mort en 1761). Il est à peu près certain que cette seconde partie, posthume, a été éditée par un groupe d'amis dont faisait partie Perronet et que c'est elle qu'il a désignée comme « nouvelle Architecture hyơraulique ". La bibliographie matérielle des ouvrages publiès sous le nom de Belidor est fort complexe, mais étant donné l'importance de la tradition technologique qu'ils représentent, il eût été utile d'éclaircir complètement le rôle de Perronet à cet égard.

Pierre Costabel.

La Figure de la Terre du XnHI siècle à l'ère spatiale. Sous la dir. de Henri Lacombe et Pierre Costabel. Paris, Gauthier-Villars, 1988. 15,3 $\times 24$, 472 p., fig. (" Académie des sciences").

Pierre Simon de Laplace écrivit : «Il est remarquable qu'un astronome, sans sortir de son observatoire, en comparant seulement ses observations à l'analyse, eût pu déterminer exactement la grandeur et l'aplatissement de la Terre, et sa distance au Soleil et à la Lune, éléments dont la connaissance a été le fruit de longs et pénibles voyages dans les deux hémisphères. L'accord des résultats obtenus par ces deux méthodes est une des preuves les plus frappantes de la gravitation universelle " (p. 308).

Peut-être se souvint-il des lignes plus poétiques et plus célèbres de Voltaire, concluant :

"Vous avez confirmé dans ces lieux pleins d'ennui,
Ce que Newton connut sans sortir de chez lui
Vous avez arpenté quelque faible partie
Des flanes toujours glacés de la terre aplatie " (p. 57).

Sans doute Voltaire favorisa trop ses amis les mathématiciens; en effet, l'étude de la figure de la terre exige encore une collaboration profonde entre les gens de 
cabinet et les gens de mesures, quoique de nos jours ces derniers rejettent « [les] perches, [les] secteurs et surtout deux Laponies " en faveur des satellites télémétriques et des Russes.

Il est facile de remarquer que, dans cette enquête, la découverte la plus saisissante, après celle de la sphéricité de la terre, est que la terre n'est pas du tout une sphère géométrique. Ici la France du $x \mathrm{XII}^{\mathrm{e}}$ siècle mérite d'être louée à plusieurs titres : grâce à Jean Picard qui a, le premier, accompli une mesure exacte de la terre sphère, à Jean Richer qui a découvert l'anomalie de la gravité vers l'équateur et à Jean Cassini qui a vu pour la première fois la sphère aplatie de Jupiter.

Ces résultats furent dus au programme de recherches astronomiques de l'Académie royale des sciences, qui dirigea les premiers voyages scientifiques, ceux de Picard à Uraniborg (destinés à déterminer exactement la position de l'observatoire de Tycho Brahe) et de Richer à Cayenne (pour fixer la parallaxe de Mars, etc.). Ce fut encore l'Académie qui organisa deux expéditions beaucoup plus célèbres, celle de Maupertuis en Laponie (avril 1736-août 1737), et celle de Godin, Bouguer et La Condamine au Pérou (1735-1743) :

" Héros de la Physique, argonautes nouveaux,

Qui franchissez les monts, qui traversez les eaux,... »

Voltaire s'en explique d'ailleurs (Lettres philosophiques, XIV) : " A Paris, vous vous figurez la terre faite comme un melon, à Londres, elle est aplatie des deux côtés. "

En effet, deux méthodes et deux systèmes philosophiques étaient violemment en conflit. La méthode géodésique (Cassini) donnait à la terre un axe allongé ; la méthode dynamique (Huygens et Newton) donnait un axe raccourci. Mais les dynamiciens suivaient des raisonnements philosophiques très différents dont la proportion des axes polaire/équatoriale devenait ou 577/578 (Huygens, 1690) ou $689 / 692(=230 / 231$ à peu près : Newton, 1687$)$. Qui avait raison?

Pour célébrer la résolution de cette question et le commencement de l'ère classique de la géodésie comme de l'étude des anomalies gravitationnelles de la terre, l'Académie des sciences organisa donc ce colloque, intitulé La Figure de la Terre du xйIf siècle à l'ère spatiale et tenu au Palais de l'Institut du 29 au 31 janvier 1986. Il fut conçu en trois parties, conformément aux vœux des ambassadeurs des pays historiquement liés à la France par ces missions scientifiques d'il y a 250 ans. Deux communications préliminaires traitent l'histoire du problème de la figure de la terre, l'une de 1670 à 1957 (J.-J. Levallois), l'autre depuis 1957, correspondant à l'ère des satellites et des ordinateurs (J. Kovalevsky). Dans la seconde partie (p. 97-319, donc la plus importante du livre), on trouve des études historiques des expéditions de 1735 à 1743 (par P. Costabel et R. Taton); également des contributions sur les instruments dont se servaient les savants français et espagnols ; sur les techniques mathématiques nécessaires pour corriger et réduire en théorie les mesures faites dans ces "lieux pleins d'ennui " respectivement polaires et équatoriales; sur l'extension du travail des "Héros" et l'origine de l'étalon métrique comme mesure naturelle dérivée de la géodésie. Enfin, la troisième partie (p. 323-448) renferme des essais sur les techniques 
modernes relatives à la figure de la terre : mathématiques, gravimétriques, télémétriques, interférométriques.

Il est difficile de caractériser une cuvre d'une telle érudition à laquelle ont participé vingt-quatre auteurs distingués. On regrettera quelques répétitions et, étant donné le plan du colloque, une certaine lacune historique pour la période allant de la Révolution (le système métrique) jusqu'à l'ère spatiale. Bien sûr, on y rencontre les noms de Gauss, de George Darwin, de Harold Jeffreys, de Radau, etc., mais le point de vue historique diminue beaucoup après l'essor géodésique de la France, situé entre 1670 et 1800 environ. Cela dit, le lecteur non spécialiste $y$ trouvera une synthèse très complète et très exacte d'un sujet qui ne possédait certes pour Voltaire qu'un intérêt philosophique, même un peu gratuit, mais qui a gagné pour nous une importance tant industrielle que militaire.

A. Rupert HALL.

Nicole Hulin-Jung, L'Organisation de l'enseignement des sciences: la voie ouverte par le Second Empire. Préf. de Robert Fox. Paris, Éd. du C.T.H.S., 1989. $21 \times 27,336$ p. ( $\times$ Mémoires de la Section d'histoire des sciences et des techniques ", 6).

Cet ouvrage, issu d'une réflexion approfondie et d'une quête documentaire exemplaire, contribue d'une manière précise, nuancée et objective à une meilleure connaissance de l'enseignement des sciences dans l'enseignement secondaire français au siècle dernier.

Le cheminement du propos est lent et s'alourdit de quelques digressions plus ou moins utiles (historique de l'agrégation et de l'École normale supérieure, biographie de Fortoul...); il s'accidente également d'un trop grand nombre de citations qui fragmentent quelque peu le texte lui-même qui perd ainsi en unité et en continuité.

Mais ce labeur consciencieux a le mérite de présenter une vue d'ensemble de l'évolution de l'enseignement des sciences tout au cours du $\mathrm{XIX}^{\mathfrak{e}}$ siècle et de souligner le rôle pionnier joué par le ministre de l'Instruction publique, Fortoul, entre 1852 et 1856.

Contrairement à l'image assez négative qui fut et est encore la sienne dans la mythologie universitaire, cet ancien saint-simonien, rallié à Napoléon III, fut d'une grande activité réformatrice notamment à propos de l'enseignement des sciences avec sa célèbre "bifurcation " (distinguant une section littéraire d'une section scientifique). Ce dédoublement pédagogique qui favorise de manière indéniable l'enseignement des sciences dans les lycées et collèges s'inscrit, en fait, dans tout un courant d'idées qui depuis la fin du XvIII siècle milite pour une promotion des sciences exactes. L'auteur évoque les projets et les efforts répétés dans la première moitié du siècle pour arracher les sciences à l'oppression des humanités classiques. Mais en vain... Il fallut tout l'autoritarisme du ministre 
Fortoul pour imposer l'égalité entre sciences et lettres. Mais ce coup d'état pédagogique se heurte à la résistance du corps enseignant et la mort prématurée de son auteur en 1856 l'empêche de s'imposer définitivement. Pourtant, l'idée d'un véritable enseignement des sciences n'est pas abandonnée et Nicole Hulin retrouve l'héritage de la "bifurcation » dans la création de l'enseignement secondaire spécial par le ministre Victor Duruy en 1865 et l'émergence d'un enseignement secondaire moderne sous la Troisième République dès avant 1900.

L'étude de Nicole Hulin, complétée par un index des personnes, de nombreux tableaux et illustrations, apparait indispensable pour le sujet qu'elle traite avec tant de soin, de minutie et d'objectivité.

Paul Gerbod.

Jérôme Ramunni, La Physique du calcul: histoire de l'ordinateur. Paris, Hachette, 1989. $14 \times 23,284$ p., bibliogr., index (" Histoire et philosophie des sciences ").

L'ordinateur est au point de convergence de nombreuses inventions et découvertes dans des domaines techniques hétérogènes, dans le champ de la physique fondamentale et dans celui des mathématiques. Une histoire linéaire, celle des générations d'ordinateurs, est ici récusée parce qu'il est tenu compte de l'environnement technique à différentes époques, des interactions entre la science fondamentale et les techniques, entre la physique théorique et expérimentale et les mathématiques, en bref de plusieurs courants de recherche, qui tous ne concernaient pas ce que l'on appellera en français "l'ordinateur" (par exemple, l'importance des télécommunications et de la cryptographie, la nécessité du comptage dans la physique subatomique et, avant cela, l'automatisation des calculs relevant de l'analyse harmonique de Fourier dans la physique du continu).

Avec cette ambition, il fallait faire des choix et le lecteur ne pourra s'étonner si l'auteur, à diverses reprises, signale les histoires qu'il n'écrira pas. Instrument de comptage et de calcul au sens ordinaire, l'ordinateur, dès le départ, est conçu aussi comme un outil pour les sciences les plus formalisées lorsque, par exemple, il n'existe pas de solution analytique à des équations que l'on considère adaptées à la mathématisation. L'ordinateur, instrument des mathématiciens (pas seulement), s'appropriera comme d'autres domaines techniques, les découvertes de la physique du discontinu en électronique et en physique des solides, et son architecture se transformera avec ces découvertes et avec l'immense travail sur la programmation et les logiciels.

Le rôle des mathématiciens et des physiciens mathématiciens ne saurait être minimisé. Tout d'abord, certains mathématiciens pensent que les mathématiques, à des moments cruciaux, tirent leur inspiration des sciences de la nature (l'approche " bottom up »), étant entendu qu'ils distinguent la recherche en mathématique de la recherche en physique théorique. Les modèles mathématiques et leur 
calculabilité sont décisifs pour les domaines de la physique les plus développés et il existerait une parenté profonde entre analyse mathématique et analyse physique d'un phénomène. La théorique physique est, dans cette optique, inséparable de la mathématique utilisée (l'idée est ancienne, qu'on pense à Fourier) et pour V. Bush en 1936, par exemple, les caicuis qui dérivent de celle-ci occupent un rôle équivalent à celui de l'instrumentation scientifique. Ce point de vue donne un ton à certains développements du livre et il évoque le problème de l' " adéquation ", souvent discutée, de la mathématique modélisatrice (cf. les débats actuels sur l'Intelligence Artificielle). On comprend alors qu'apparaissent, dans une histoire de l'ordinateur, les noms de savants qui ont apporté une contribution à la modélisation, ce qui pose le problème du calcul et de l'architecture la plus adaptée à cet objectif. Au XIX ${ }^{e}$ siècle, il faut automatiser les calculs de l'analyse de Fourier et Lord Kelvin mettra au point le premier calculateur analogique. Au $x x^{e}$ siècle, von Neumann travaillera sur l'axiomatisation de la mécanique quantique et sur la théorie des jeux et se poseront alors à lui et à ses collègues de redoutables problèmes de modélisation; il sera l'un des concepteurs de la machine dotée d'une structure qui porte son nom.

L'ordinateur n'est évidemment possible que grâce aux avancées techniques. Si les machines analogiques sont limitées à une certaine mathématique, si tout système formel peut être arithmétisé, alors, avec les découvertes de matériaux fiables (problème de l'erreur), la miniaturisation, une grande capacité de mémoire et une très grande vitesse de calcul, le numérique remplace l'analogique. L'électronique se substituera à l'électro-mécanique, les tubes à vide, fragiles, seront remplacés par les semi-conducteurs, importants aussi pour les télécommunications, le radar... Il existe un prototype complètement transistorisé en 1954, ce qui fait qu'au plan non commercial la deuxième " génération » vient avant la première. Les tores en ferrite équiperont la mémoire centrale, puis on pensera à eux pour la partie logique et mathématique et, dès 1957 , on aura une machine faisant la synthèse entre transistors et tores en ferrite. A noter qu'il existe une richesse d'idées, de prototypes qui n'aboutiront pas à des produits commerciaux essentiellement pour des raisons économiques: les machines totalement en tores en ferrite, l'ordinateur supraconducteur... Ce ne sont évidemment pas seulement des exploits d'ingénieur : les idées et réalisations techniques sont sous la dépendance des connaissances fondamentales acquises dans les laboratoires (physique du solide, supraconductivité...). Les matériaux utilisés dans ces machines à structures complexes permettront une plus grande rapidité de calcul et une miniaturisation. On aura les circuits imprimés puis intégrés (on passera d'un composant par millimètre carré à vingt mille), qui auront un retentissement sur l'architecture de l'ordinateur. L'organisation hièrarchique linéaire de von Neumann avait èté mise en cause par des mathématiciens et physiciens, par les spécialistes des programmes, mais l'intégration conduira à l'essor des réseaux d'ordinateurs sur la base d'une réflexion portant sur la topologie des interconnexions.

L'ordinateur, c'est aussi la programmation. Très vite les ingénieurs, les commerciaux, se préoccuperont de rendre compatibles des matériels différents. Beaucoup de langages seront mis au point, la programmation étant traduite en langage machine et stockée en sous-programmes sur des mémoires auxiliaires (FORTRAN, 
mis au point pour les premières machines séquentielles, sera adapté aux machines parallèles). Lorsque les ordinateurs deviendront plus rapides, plus complexes, il faudra gérer cette complexité grâce aux logiciels afin d'en optimiser l'utilisation. La multi-programmation permettra de partager un ordinateur entre plusieurs usagers, de manière simultanée.

Se rejoignent donc la technique, les mathématiques et la mise en œuvre du logiciel, ce qui, à terme, donne raison à Türing (1945) qui avait très tôt perçu que le programme commande le choix des circuits, que le matériel est indissolublement lié au logiciel, ce qui l'avait conduit à une machine à structure feuilletée diffërente de celle de von Neumann.

Dans cette histoire complexe, mentionnons encore le souci des constructeurs de dissimuler à l'utilisateur, grâce aux langages évolués, la complexité de la machine. Ne sont plus alors requis des programmeurs de très haut niveau, ce qui permet, en conjonction avec d'autres facteurs, la diffusion des micro-ordinateurs, la mise au point des systèmes-experts et conduit aussi à des discussions passionnées sur l'Intelligence Artificielle.

Les ordinateurs sont très vite sortis du laboratoire. Ils ont été adoptés par les compagnies d'aviation, d'assurances, par le secteur commercial et, plus généralement, le tertiaire. La "révolution " informatique c'est, en partie, cet immense marché avec la micro-informatique professionnelle (familiale et scolaire aussi), les réseaux et la télématique. On comprend que les stratégies commerciales et les besoins des clients ont pesé dans un certain nombre de choix techniques qui devaient conduire, entre autres choses, au tout numérique.

L'auteur insiste à plusieurs reprises sur le rôle des militaires. L'Armée (en particulier américaine) est partout présente dans ce livre. De quoi a-t-on besoin pour la défense, le radar, les satellites, les missiles, etc. ? Que faut-il faire pour ne pas se laisser dépasser par les Soviétiques, pour progresser dans la guerre des étoiles? Les investissements sont à la mesure des volontés stratégiques, des inquiétudes et peurs provoquées par le géant de l'Est. Notons aussi l'étroite coopération entre les laboratoires universitaires et ceux de l'Armée, ce qui nous vaut des réflexions dont tireront profit ceux qui s'intéressent aux relations entre science, technique et société.

Nous avons là un ouvrage intelligent, superbement documenté, trop bref pour l'ambition de l'auteur qui, visiblement, aurait aimé approfondir certaines analyses qui ne sont qu'esquissées et qui, espérons-le, seront reprises dans d'autres textes.

Gérard Lemaine. 